\title{
LOCAL CROSS SECTIONS IN LOCALLY COMPACT GROUPS
}

\author{
PAUL S. MOSTERT ${ }^{1}$
}

Introduction. Let $p$ be the natural projection of the topological group $G$, with subgroup $H$, onto the coset space $G / H$. The subgroup $H$ is said to have a local cross section if there exists an open set $U$ in $G / H$, and a continuous function $f$ defined on $U$ with values in $G$ such that $p f(x)=x$ for $x$ in $U$. The most general conditions on $G$ and $H$ under which such a function exists are not known. It has been conjectured $[7, \text { p. 33 }]^{2}$ that if $G$ is compact and of finite dimension, then $H$ has a local cross section. (For the infinite-dimensional case, there are examples of compact groups with closed subgroups not having a local cross section.) In this paper, we show that if $G$ is locally compact, separable, metric, and of finite dimension, and $H$ is a closed subgroup of $G$, then $H$ has a local cross section. In $\$ 1$, several elementary lemmas necessary for the proof are stated, along with certain properties of Lie groups and projective limits. In $\$ 2$, we prove the main theorem.

1. Preliminary definitions and theorems. We shall, in the following, use, principally, the notation and terminology of [4]. Let $\left\{G_{k}\right\}$ be a sequence of groups indexed by the positive integers. Suppose there exists, for each $k$, a continuous, open homomorphism $\pi_{k}^{k+1}$ of $G_{k+1}$ onto $G_{k}$. Let $G^{*}=P_{k} G_{k}$. Then the group $G=\left[x=\left\{x_{k}\right\} \mid \pi_{k}^{k+1}\left(x_{k+1}\right)=x_{k}\right]$ is called the projective limit of the sequence $\left\{G_{k}\right\}$ and $\pi_{k}$ is the projection of $G$ on $G_{k}$. The following properties of projective limit groups may be found in [4, pp. 54-56], [2, pp. 212-232], or else are easily verified.

LemMa 1. If $H$ is a closed subgroup of $G$, where $G$ is the projective limit of Lie groups, and $H_{k}$ is the natural projection $\pi_{k}(H)$ in $G_{k}$, then $H$ is the projective limit of $\left\{H_{k}\right\}$, and $G / H$ is the projective limit of $\left\{G_{k} / H_{k}\right\}$.

$G^{*}$ has the usual Tychonoff topology. $G$ is a closed subgroup of $G^{*}$ under the induced topology. An open subset in $G$, then, contains an

Presented to the Society, September 4, 1952; received by the editors August 15, 1952 and, in revised form, December 13, 1952.

1 The author wishes to express his appreciation to Professor M. E. Shanks for valuable suggestions and criticisms which he offered in the preparation of this paper, and to the referee for observing that the proof was valid for a much wider class of groups than was originally supposed.

2 Numbers in brackets refer to the references at the end of the paper. 
open set of the form $G \cap P_{k} W_{k}$ where

$$
W_{k}=\left\{\begin{array}{l}
V_{k} \text { open in } G_{k}, \quad k=k_{1}, \cdots, k_{n}, \\
G_{k} \text { otherwise. }
\end{array}\right.
$$

Let $\left(\pi_{k}^{k+1}\right)^{-1}=\pi_{k+1}^{k}, \pi_{k}^{k+1} \cdots \pi_{k+m-1}^{k+m}=\pi_{k}^{k+m}, \pi_{k}^{k}=$ identity map in $G_{k}$. Define

$$
U_{k}=\bigcap_{m=k_{1}}^{k_{n}} \pi_{k}^{m}\left(W_{m}\right)
$$

Then $G \cap P_{k} U_{k}=G \cap P_{k} W_{k}$. Similar open sets are in $G / H$.

TheOREM 1. Let $G$ be the projective limit of the sequence $\left\{G_{k}\right\}, H a$ closed subgroup of $G, H_{k}=\pi_{k}(H)$, and $\tilde{\pi}_{k}^{k+1}$ the induced mapping $G_{k+1} / H_{k+1} \rightarrow G_{k} / H_{k}$. Then a sufficient condition for $H$ to have a local cross section is that for some open set $U=(G / H) \cap P_{k} U_{k}$, as defined above, there exist local cross sections $f_{k}: U_{k} \rightarrow G_{k}$ such that $\pi_{k}^{k+1} f_{k+1}=f_{k} \bar{\pi}_{k}^{k+1}$.

Proof. Define $f\left(\left\{x_{k}\right\}\right)=\left\{f_{k}\left(x_{k}\right)\right\}$. One may easily verify that $f$ satisfies the conditions of a local cross section.

LEMMA 2. Let $G_{1}, G_{2}$ be two Lie groups (of the same dimension), $\pi$ a continuous, open homomorphism, with finite kernel, of $G_{2}$ onto $G_{1}$. If $V_{1}$ is an open $n$-cell neighborhood of the identity e in $G_{1}$, then there exists $a$ set $V_{2}^{e}$ homeomorphic, under $\pi$, with $V_{1}$, and if $\pi^{-1}(e)=\left[s_{1}, \cdots, s_{m}\right]$,

$$
V_{2}=\pi^{-1}\left(V_{1}\right)=\bigcup_{i=1}^{m} s_{i} V_{2}^{*}
$$

Lemma 3. Under the hypothesis of Lemma 2, if $\mathrm{H}_{2}$ is a closed subgroup of $G_{2}, H_{1}=\pi\left(H_{2}\right), U_{1}=p_{1}\left(V_{1}\right), U_{2}^{e}=p_{2}\left(V_{2}^{e}\right)$, where $p_{i}$ is the natural projection $G_{i} \rightarrow G_{i} / H_{i}$, then $U_{1}$ is homeomorphic with $U_{2}^{e}$.

Lemmas 2 and 3 are straightforward and easy in proof.

2. Principal results. In this section, all groups are assumed to be separable, metric groups.

TheOREM 2. If $H$ is a closed subgroup of a 0 -dimensional compact group $G$, then $H$ has a cross section.

A cross section of $H$ is a local cross section for which $U=G / H$. From $[7$, pp. 31 and 36], we have the following consequence:

COROLLARY. Under the hypothesis of Theorem 2, $G$ is $H$ equivalent to the product bundle $H \times G / H$.

Proof of Theorem 2. $G$ is the projective limit of the sequence 
$\left\{G_{k}\right\}$ of finite groups. $G_{1}=\left[s_{1}^{1}, \cdots, s_{m_{1}}^{1}\right]$ where we assume $s_{1}^{1}, i$ $=1, \cdots, m_{1}$, are distinct $\bmod H_{1}$, and are isomorphic to $G_{1} / H_{1}$. (See Theorem 1 for the definition of $H_{k}$.) The set $\left[s_{1}^{1}, \cdots, s_{m_{1}}^{1}\right]$ is a cross section $f_{1}$ of $H_{1}, G_{2}=\left[s_{1}^{2}, \cdots, s_{n_{2}}^{2}\right]$ where we order the elements so that $\pi_{1}^{2}\left(s_{i}^{2}\right)=s_{1}^{1}$ for $i=1, \cdots, m_{1}, s_{1}^{2}, \cdots, s_{m_{2}}^{2}, m_{2} \geqq m_{1}$, are distinct $\bmod H_{2}$, and are isomorphic to $G_{2} / H_{2}$. This is possible since $\pi_{1}^{2}\left(H_{2}\right)$ $=H_{1}$. Then the set $\left[s_{1}^{2}, \cdots, s_{m_{2}}^{2}\right]$ is a cross section $f_{2}$ of $H_{2}$ which agrees (in the sense of Theorem 1 ) with $f_{1}$. Continuing this process, we obtain cross sections satisfying the hypothesis of Theorem 1.

LEMMA 4. Let $G$ be a locally compact group which is the projective limit of the Lie groups $\left\{G_{k}\right\}$, where $G_{k}=G / N_{k}, N_{k}$ a compact normal subgroup of $G$. Then $\pi_{k+1}^{k}(e)$ is compact.

Henceforth, we shall mean projective limit in the sense of Lemma 4 when we say simply "projective limit."

TheOREM 3. Let $G$ be a locally compact group of finite dimension, $H$ a closed subgroup of $G$. Then $H$ has a local cross section.

Proof. We may assume, because of the results of Gleason [3] and Montgomery-Zippin [5], that $G$ is the projective limit of Lie groups. Also, these groups may be assumed to be of the same dimension (see $\left[6\right.$, p. 214]). Hence, $\pi_{k+1}^{k}(e)$ is finite for every $k$ by Lemma 4 . We shall construct local cross sections satisfying the hypothesis of Theorem 1.

Let us keep in mind the following diagram:

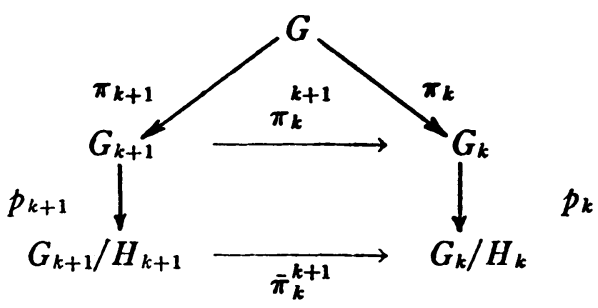

There exists an open set $U_{1}^{\prime}$ in $G_{1} / H_{1}$ containing $H_{1}$, on which a local cross section is defined. ( $H_{1}$ is closed if $H$ is, so the above statement follows from [1, Proposition 1, p. 110].) Let $V_{1}$ be an open $n$-cell neighborhood of the identity which is contained in $p_{1}^{-1}\left(U_{1}^{\prime}\right)$. Construct neighborhoods $V_{k}=U_{i=1}^{k} s_{k}^{k} V_{k}^{e}, k>1$, by Lemmas 2 and 3, where $\left[s_{1}^{k}, \cdots, s_{n_{k}}^{k}\right]=K_{k}=\pi_{k}^{1}(e)$, the $s_{t}^{k}$ being ordered as below. Let $\theta_{k}$ be the homeomorphism of $V_{1}$ onto $V_{k}^{e}$ (see Lemma 2) induced by $\pi_{1}^{k}$, and $\bar{\theta}_{k}$ be the homeomorphism, induced by $\theta_{k}$, of $U_{1}$ onto $U_{k}^{e}$ $=p_{k}\left(V_{k}^{e}\right)$. We order the $s_{i}^{2}$ as follows: $s_{i}^{2}$ are distinct $\bmod H_{2}$ for $i=1, \cdots, m_{2} \leqq n_{2}$, and are isomorphic to the set of left cosets of $H_{2}$ in 
$K_{2} H_{2}$. Then ${ }^{8}$

$$
\bar{\pi}_{2}^{1}\left(U_{1}\right)=\bigcup_{i=1}^{m_{2}} s_{i}^{2} U_{2}^{e}=\bigcup_{i=1}^{m_{2}} p_{2}\left(s_{i}^{2} p_{2}^{-1}\left(U_{2}^{e}\right)\right)=\bigcup_{i=1}^{m_{2}} p_{2}\left(s_{i}^{2} V_{2}^{e}\right)
$$

Define $f_{2}$ on $U_{2}^{e}$ by

$$
f_{2}(x)=\theta_{2} f_{1} \tilde{\pi}_{1}^{2}(x) \text {. }
$$

Since $s_{i}^{2} U_{2}^{e}$ is disjoint from $s_{1}^{2} U_{2}^{e}$ if $i \neq j, i, j \leqq m_{2}$, we may define $f_{2}$ on $s_{1}^{2} U_{2}^{e}$ as

$$
f_{2}\left(s_{i}^{2} x\right)=s_{i}^{2} f_{2}(x) \text {. }
$$

$f_{2}$ is obviously continuous. That it satisfies the conditions of a local cross section is immediate from $p_{2}=\bar{\theta}_{2} p_{1} \pi_{1}^{2}$ on $U_{2}^{e}$, and

$$
p_{2}\left(s_{i}^{2} f_{2}(x)\right)=s_{i}^{2} p_{2}\left(f_{2}(x)\right)=s_{i}^{2} x
$$

Also

$$
\pi_{1}^{2} f_{2}\left(s_{i}^{2} x\right)=\pi_{1}^{2}\left(s_{i}^{2}\right) \pi_{1}^{2} f_{2}(x)=\pi_{1}^{2} f_{2}(x)=\pi_{1}^{2} \theta_{2} f_{1} \bar{\pi}_{1}^{2}(x)=f_{1} \bar{\pi}_{1}^{2}\left(s_{i}^{2} x\right) .
$$

We now order $K_{3}$. Choose $s_{i}^{8}, i=1, \cdots, m_{2}$, so that $\pi_{2}^{3}\left(s_{i}^{3}\right)=s_{i}^{2}$ and $s_{i}^{8}, i=1, \cdots, m_{3}, n_{3} \geqq m_{3} \geqq m_{2}$, are in different left cosets of $H_{3}$, and are isomorphic to the set of left cosets of $H_{3}$ in $K_{3} H_{3}$. This we can do since $\pi_{3}^{2}\left(K_{2}\right)=K_{3}$. The $s_{i}^{3}$ may be chosen arbitrarily for $i>m_{3}$. Define the local cross section $f_{3}$ on $U_{3}^{e}$ by

$$
f_{3}(x)=\theta_{3} f_{1} \bar{\pi}_{1}^{3}(x),
$$

and similar to $f_{2}$ on $s_{i}^{3} U_{3}^{e}$ for $i=1, \cdots, m_{3}$. Then $f_{3}$ is a local cross section on $\bar{\pi}_{3}^{1}\left(U_{1}\right)=\bar{\pi}_{3}^{2}\left(U_{2}\right)$, where

$$
U_{2}=\bigcup_{i=1}^{m 2} s_{i}^{2} U_{2}^{e}
$$

and

$$
\begin{aligned}
{\stackrel{\pi}{2} f_{3}\left(s_{i}^{3} x\right)}^{3} & =\pi_{2}^{3}\left(s_{i}^{3}\right) \pi_{2}^{3}\left(f_{3}(x)\right)=s_{i}^{2} \pi_{2}^{3} \theta_{3} f_{1}\left(\dot{\pi}_{1}^{3}(x)\right) \\
& =s_{i}^{2} \theta_{2} f_{1}\left(\bar{\pi}_{1}^{3}(x)\right)=s_{i}^{2} f_{2} \bar{\theta}_{2} \bar{\pi}_{1}^{8}(x)=s_{i}^{2} f_{2}\left(\bar{\pi}_{2}^{3}(x)\right) \\
& =f_{2}\left(s_{2}^{3} \bar{\pi}_{1}^{2}(x)\right)=f_{2} \bar{\pi}_{2}^{3}\left(s_{i}^{3}(x)\right) .
\end{aligned}
$$

We order $K_{4}$ relative to $K_{3}$ in the same manner as we did $K_{8}$ relative to $K_{2}$, and make a similar definition of the local cross section

${ }^{3}$ For $s$ in $G$ and $x$ in $G / H$, we define $s x=p\left(s p^{-1}(x)\right)$ where $p$ is the natural projection of $G$ on $G / H$. 
$f_{4}$. Continuing this process, we obtain the desired local cross sections, and hence, by Theorem $1, H$ has a local cross section.

$G$ has an open set $W$ which is a direct product, $W=Z A$, where $Z=\pi_{1}^{-1}(e)$ is the projective limit of the groups $K_{k}$, and $A$ is a local Lie group. (See [5, pp. 214-215].) The set $U=Z V$, where $V$ is the limit of $V_{\mathbf{k}}^{e}$, is an open set contained in $W$ (or may be so chosen). The set $R=S T$, where $S$ is a cross section set of $Z / Y, Y=H \cap Z$, and $T$ is the projective limit of $f_{k}\left(U_{k}^{o}\right)$, is a cross section set of $U$ by our construction. If $H$ is closed, then $B=A \cap H$ is a local Lie group such that $W \cap H=Y B$. One may note from our construction that $T$ is a cross section set for $B$ in $A$. Hence, we have the following result.4

COROLLARY 1. Let $G$ be a group satisfying the hypothesis of Theorem 2. Let $W=Z A$, where $Z$ is 0-dimensional, compact subgroup of $G, A$ a local Lie group. If $H \cap W=Y B$ is the corresponding decomposition of the open set $H \cap W$, then $G / H$ is locally the direct product of $Z / Y$ and $A / B$.

Corollary 2. Let $G$ be a locally compact group which is separable, metric, and of finite dimension, and $H$ a closed subgroup of $G$. Then $G$ is a fibre bundle over $G / H$.

Proof. See [7, p. 31].

\section{REFERENCES}

1. C. Chevalley, Theory of Lie groups, Princeton University Press, 1946.

2. S. Eilenberg and N. Steenrod, Foundations of algebraic topology, Princeton University Press, 1952.

3. A. M. Gleason, Groups without small subgroups, Ann. of Math. vol. 56 (1952) pp. 193-212.

4. S. Lefschetz, Algebraic topology, Amer. Math. Soc. Colloquium Publications, vol. 27, 1942.

5. D. Montgomery and L. Zippin, Small subgroups of finite dimensional groups, Ann. of Math. vol. 56 (1952) pp. 213-241.

6. L. Pontrjagin, Topological groups, Princeton University Press, 1946.

7. N. Steenrod, Theory of fibre bundles, Princeton University Press, 1951.

Purdue University

- One may observe that this decomposition enables us, using Theorem 1 and Proposition 1, p. 110 of [1], to construct a local cross section for $H$ in a slightly different way. This fact and Corollary 1 were pointed out to the author by the referee. 